\title{
Towards Real-Time Strategic Teamwork: A RoboCup Case Study
}

\author{
Kenichi Yoshimura ${ }^{1}$, Nick Barnes ${ }^{1}$, Ralph Rönnquist ${ }^{3}$, and Liz Sonenberg ${ }^{2}$ \\ 1 Department of Computer Science and Software Engineering \\ The University of Melbourne, Parkville, VIC, Australia \\ $\{$ kyosh, nmb\}@cs.mu.oz.au \\ 2 Department of Information Systems \\ The University of Melbourne, Parkville, VIC, Australia \\ 1.sonenberg@dis. unimelb.edu.au \\ 3 Agent-Oriented Software Pty. Ltd. 221 Bouverie St. Carlton, VIC Australia \\ ralph.ronnquist@agent-software.com.au
}

\begin{abstract}
RooBots competed in the F180 League of the RoboCup 2001 competition in Seattle, USA. In this article, we present an architectural overview of our system involving an integration of an agent-oriented programming framework to support strategic decisions, with various lowlevel perception and control elements. Our AI Module includes a novel mechanism to facilitate dynamic formation change by an individual agent and we report a preliminary evaluation of the approach drawn from performance in the 2001 competition.
\end{abstract}

\section{Introduction}

Two University of Melbourne RoboCup teams, MU-Cows 2000 4 and RooBots 2001 [8, have participated in the F180 League. In 2000 the emphasis was on hardware and software issues at the individual robot level of action. A major advance for 2001, and the focus of this article, was the addition of an AI Module enabling the coding, and rapid modification, of high level strategies.

In this paper we motivate an agent-oriented approach to robotic soccer for hardware leagues, and address the following questions associated with the implementation of the AI Module.

1. How can one take advantage of the high-level abstractions supported by the agent-oriented approach for specifying strategic teamwork within the constraints of a real-time domain and the realities of low level robot control? (Sections 2 and 3)

2. How can one facilitate tactically appropriate, dynamic restructuring of team formations (Sections 4 and 5]?

\section{Architectural Overview}

Our base system SmHost has a modular architecture, with interactions among the modules specified by interface definitions. The architecture proved highly 


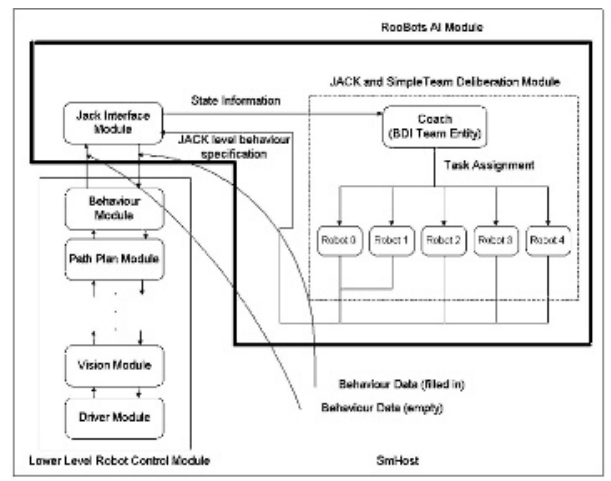

Fig. 1. Architectural Overview of SmHost and AI Module.

successful in facilitating modification of the base system: easy customisation for different hardware configurations (eg. a new path planner for omnidirectional robots), reusing existing components (eg. the vision system), and development of new modules (eg. the AI module). The resulting system used in 2001 is illustrated in Figure 1

In the F180 League a camera is mounted above the field, enabling teams to perceive most required information with each frame. Objects are identified by their colours, and positions of the objects are calculated using camera calibration with associated uncertainty.

The Behaviour Module interacts with the Path Planning Module to implement basic skills such as kicking, dribbling, marking of an opposition robot, moving to a point, and goalkeeper behaviour. The behaviours are robust in the face of failures. For example, once it commits to a particular behaviour, a robot pursues a given goal until a new behaviour is selected. We use a method that combines deliberative and reactive path planning to plan a collision-free path. The Behaviour Module specifies a destination anywhere within the field and the robot moves to it efficiently without collisions.

\section{The AI Module}

The behaviour of an agent depends on its role. We used two distinctive roles for field players - a defensive and an offensive role - and the specialised goalkeeper. Role assignment occurs by manual setting based on formations (Section [3.3) but can be overridden by other run-time considerations (Section 4). Behaviour specification occurs at a strategic level (Section 3.4 both for individual agent behaviour, and team-level coordination.

\subsection{Module Overview}

The module has two major components - the Deliberation Module and the Jack Interface Module. The Deliberation Module comprises agents representing in- 
dividual robots and a team entity (the Coach) responsible for coordination of individual activities. It is implemented using an agent-oriented programming environment, as discussed in Section 3.2. While providing programming abstractions suitable for representing strategic behaviour, the relatively low speed of this module introduced computational overheads that were unacceptable for the high-speed, real-time robotic domain. To overcome the problem of the inherited computational overhead of the agent-oriented programming framework, the deliberation process of the AI Module is supported by a faster, short-term goal selection module - the Jack Interface Module, which is invoked at the same frequency as the rest of the system. It performs short-term goal selection based on the instructions, ie. partial plans which require further deliberation before being mapped to one of the behaviours provided by the Behaviour Module.

\subsection{The Agent-Oriented Programming Environment}

JACK Intelligent Agents has been developed by Agent Oriented Software Pty. Ltd. 1] to provide general-purpose agent-oriented extensions to the Java programming language. The theoretical Belief Desire Intention (BDI) model of artificial intelligence was used as a foundation of the JACK framework. JACK agents are autonomous software components that have explicit goals to achieve or events to handle (desires). Each agent holds a plan library, i.e. a collection of plans that provide sequences of instructions to achieve a goal or handle a given event. Plans can be abstract, or partial, requiring completion at run-time. The agent pursues its given goals, adopting the appropriate plans (intentions) according to its current set of data (beliefs) about the state of the world.

SimpleTeam [39] is a plug-in extension of JACK Intelligent Agents. It provides an abstraction that separates the specification of team-level strategies and the specification of individual agents' activities. This was achieved by the introduction of a new software entity, a team instance. A team instance is the software representative for a team, and it exists to coordinate behaviour and facilitate agent communication.

In SimpleTeam, a system is modelled in terms of participating agents (teams). A programmer can specify a particular type of team instance by declaring what constitutes a team in terms of roles, eg. an attacking team consists of left wing role and right wing role. Furthermore, a role only specifies the requirements to take on the role, rather than specifying the actual teams (agents). That is, team members can be selected dynamically at run-time depending on the availabilities of the agents. The instance of the team is used to monitor team progress, and coordinate activities among the team members as required.

Team instances can also be compared with the so-called facilitators used in multi-agent systems (see, for instance, Open Agent Architecture 2]). However, while facilitators normally provide only a communications and brokering facility, team instances actually perform all reasoning related to coordination and distribution of activity among members, which provides facilities for specification of teams with coordination of joint activities among the team members. 


\subsection{Formations, Roles, and Zone-Based Distribution of Tasks}

We started our design and implementation by using the previously studied notion of formations, roles and zones to develop our team [6]. A formation is a representation of overall team strategy, summarising the number of defenders and attackers. A role is an explanation of responsibilities of an individual player (ie. one of defender, attacker or goalkeeper). For example, a player taking on a role attacker aims to score a goal while considering the best course of action to make the contributions to the team. A zone is a portion of the field for which a player is responsible or allowed to reallocate itself freely. A different team formation can be selected from the graphical user interface of the system.

\subsection{High Level Behaviours}

The AI Module consists of: the Jack Interface Module that connects the highlevel (strategy) with appropriate low-level behaviours (motion) (Section 2); five agents, each an ordinary BDI agent that determines the behaviour of an individual robot based on its current role, as determined by the initial formation (Section 3.3) or other factors (Section 4); and a SimpleTeam team entity (the Coach), responsible for role assignments and coordination of individual agent activities. The Coach performs a role assignment depending on the current team's formation and availability of the agents in the system (ie. a robot can be removed from the field due to hardware problems or being penalised by the referee). The Coach posts an appropriate event to the individual agent in the system when new state information arrives (eg. defender event for a defensive agent).

An individual agent decides what to do depending on its current role, which in turn is specified by various TeamPlans of the Coach team entity. An example is shown in the following statements of the TeamPlan perform role assignments. The first statement posts a defensive event to robot 1 , and the second statement posts an offensive event to robot 2. A different combination of those specifications allows a designer to develop a new team strategy without having to specify low level behaviours.

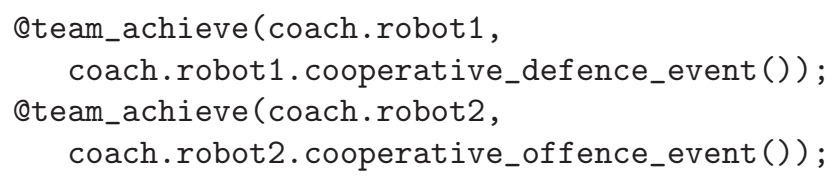

Qteam_achieve (coach.robot1,

coach.robot1.cooperative_defence_event ());

Qteam_achieve(coach.robot2, coach.robot2.cooperative_offence_event());

This modular separation of high-level and lower-level behaviours allowed rapid modification of the team-level strategy (by modification of TeamPlans for Coach team entity), and modifications of the individual agent behaviours (by modification of Plans for the individual BDI agent).

A new team strategy can be integrated into the system by writing a TeamPlan for the Coach team entity (ie. invoking a different combination of behaviours by the individual agent). The new plan can be added to the plan library of the Coach team entity without modifying any other components of the system. 
Below is a selection of the partial plans available to the deliberation components (JACK-level behaviours). For example, the Attacker Behaviour involves deliberation to pass the ball to a team member when the agent is not in a position to take a direct shot at the opponent goal. An identification of a pass receiver is done in the Jack Interface Module as the location of the receiver is constantly changing.

- DEFENCE CLEAR BALL BEHAVIOUR: The robot attempts to clear the ball from the team's defence zone. It attempts to pass the ball to the closest attacker (a robot located in the opponent defence zone) or kick the ball towards opponent goal. If the ball is located too close to the own goal, it dribbles the ball away from the goal before it attempts to kick the ball.

- ATTACKER BEHAVIOUR: Behaviour of forward player. If it has possession of the ball, it attempts to shoot a goal, pass the ball to team members, or dribble to a better position, depending on the distance between the current location and the opponent goal. If it does not have ball possession, it attempts to move to an open space and wait for a pass from team members.

\section{Dynamic Restructuring of Formations}

Stone and his colleague achieved flexibility by switching the team's formation dynamically using globally accessible information such as a time remaining and a current score difference. The triggering conditions for a dynamic formation change was specified in Locker-room agreements [6]. We adapted Parker's idea of motivation [5] for the RoboCup domain to introduce a different form of flexibility to the formation, role and zone based approach. In Parker's work, an individual robot takes its progress (ie. relative performance of the task), and the progress of other robots, into account when it makes a decision regarding to which task the robot should commit. Rather than modelling the motivation using relative performance, which seems impossible to obtain meaningfully in this domain, we modelled the urgency of the situation.

The urgency motivations utilise information such as number of team members in defence and offence zone, distance between player and ball, impatience, and defensiveness and offensiveness factors. The approach proved to be useful in the domain as it allowed dynamic restructuring of the current formation, in which an individual agent decides to disobey the current formation and role specifications, and participate in an activity that requires an immediate attention. The defensiveness and offensiveness factors express how much risk the team is willing to take in order to gain potential benefits (eg. when the offensiveness is set to zero, the team is not offensive at all. Consequently, a defensive agent would never consider participating in an offensive activity). Those values can be modified through the graphical user interface of the AI Module.

In summary, there were two distinctive roles within the system: a defensive role and an offensive role. Depending on the role an agent is playing, the contribution made by the agent differs. In principle, the formation specification assigns 
a role to an individual agent. Depending on a result of an individual agent's urgency calculation, it can either decide to play the specified role, or another role that appears more urgent. Although the urgency calculation allows an individual agent to decide whether or not to obey its primary role specification (ie. specified by a current formation), the agent remembers its primary role even if it decides to disobey the role temporarily. That is, once an urgent situation ends, the agent returns to its primary role. Hence, the current formation of the team and the results of the urgency calculations by the individual agents determine the number of agents playing a particular role.

\section{Evaluation}

The key question we would like to answer is: can (relatively abstract) strategic behaviours be specified in the deliberation component (as a combination of individual and team behaviours) in a way that robust and effective team play occurs, when the strategy is elaborated in context at run-time, and then mapped to low level behaviours. A thorough evaluation of the effectiveness of the combination of the various elements integrated into our system would require extensive empirical work, in both 'set plays' and real game situations.

In the following evaluations, we explore the added flexibility in dynamic restructuring that may be offered by allowing the urgency calculations to override the manual assignment to attacker or defender roles. Further analysis and evaluation of the system is reported in [10].

For the purpose of this analysis, we used videos of footage taken from the overhead game camera. The output of the videos can be fed into the system in the same way as the real game setting, and the system is able to perform analysis of the current situation in the same way except it cannot actually control the robots, ie. the system assumes video output is the actual image taken from the overhead camera, and performs analysis to prepare instructions for the robots. Although it does not allow evaluation of every individual aspect of the system in every possible scenario, it does allow us to see how the system really performs given the situations that arise in the tournament.

The video can also be used to identify and comment on the strengths of particular aspects of performance against a real competitor. This is in some ways better than just setting up own experiments as it shows fitness for purpose to an external goal, rather than just in a contrived situation.

Although the game against RoGi ended as a scoreless draw, it is an interesting game for post-game analysis because the RooBots did not create enough opportunities to score goals during the game. There were number of occasions when additional robots in the offensive activities could have increased the chance of scoring goals against them because RoGi was playing very defensively. Figure 2 illustrates a typical situation which was observed frequently during the game.

Figure 3 illustrates the motivation of defensive agent $D_{1}$ during the game against the RoGi Team. The result suggests there were several occasions in 


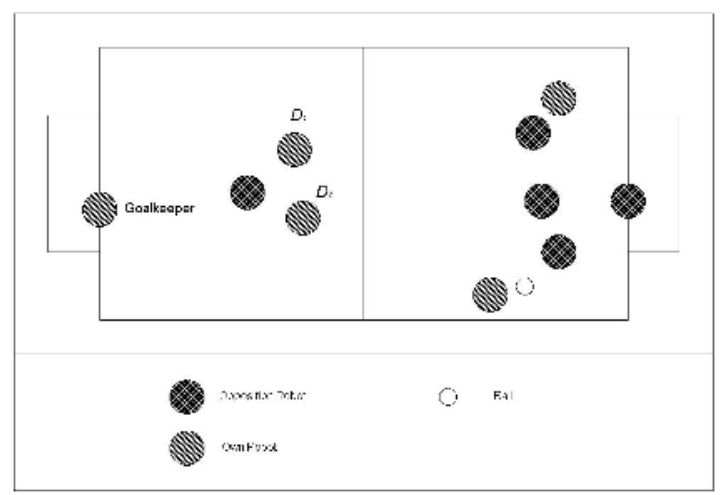

Fig. 2. RoGi Team's very defensive strategy made RooBots offensive robots to score a goal very difficult.

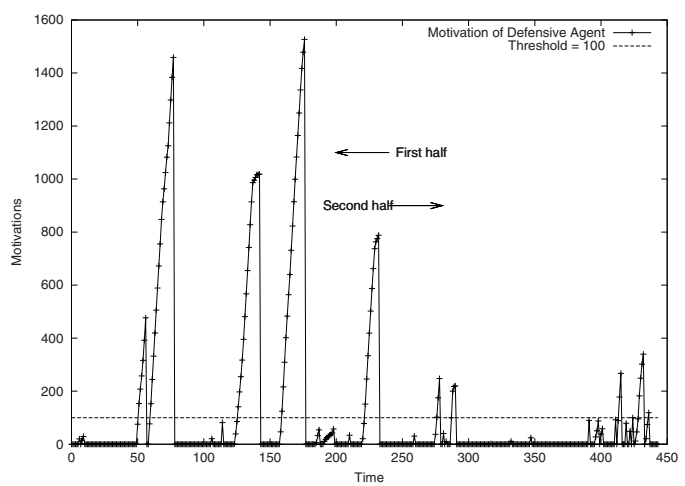

Fig. 3. Motivation of defensive agent $D_{1}$ during the game against RoGi illustrating threshold-dependent potential role shifts.

which the agent could have been eager to participate in the offensive activity, ie. the value of motivation exceeded the threshold value.

A comparison of the motivation in the first half and the second half suggests RoGi changed their strategy in the second half of the game, because the increase in the agent's motivation was much greater in the first half (maximum value of 1500) while the value was much less in the second half (maximum value of 340 ). The comparison also suggests it was a more dynamic game in the second half because urgent situations are resolved much faster - hence the maximum value of the motivation was less.

Post-game analysis of the game against RoGi revealed the most opportunities for the urgency calculation to have an impact. However the post-game analysis of the game against Cornell also deserves comment. Both Cornell and RooBots had reliable and fast omnidirectional robots, and demonstrated a number of strategic plays. Of particular note is the cooperative defensive strategy of the 
RooBots' team, discussed further in 10]. The defensive robots were marking the opposition robots cooperatively, which minimised the efficiency of Cornell's passing game, and kept direct shots on goal to a minimum. The defensive agents switched their activities flexibly among themselves, for example, they identified unmarked opposition players to mark, and demonstrated emergent cooperative strategic placement to minimise goal scoring (eg the second robot places itself behind the defensive robot located closest to the ball). Cornell Big Red scored most of their goals when the RooBots' team was penalised near the goal.

\section{Discussion}

Roobots played competitively in the 2001 competition. The AI Module was developed using a general-purpose, agent-oriented programming environment that facilitated specification, and rapid modification of strategic behaviours at an abstract level that was elaborated, in context, at run-time. To manage realtime operation while allowing adequate time for deliberation, timing issues were handled by coding at an intermediate level and by adopting a rapid computation to guide role switching and team restructuring.

Preliminary evaluation of the addition to the AI module of the local computation of heuristics to facilitate timely role changes, has demonstrated the viability of the approach to impact on the play of the game, even though this approach was not able to be tested in live games. Our ability to encode and rapidly adapt both individual and team behaviour at the strategic level during the competition was a clear demonstration of the value of high-level, agent oriented, programming. Areas of attention for future work include: additional knowledge engineering to encode a richer range of behaviours; and further exploration of ways to enhance dynamic role allocation and team restructuring.

\section{Acknowledgments}

The authors would like to acknowledge the effort and involvement of the RooBots 2001 team. In particular, we would like to express our appreciation to Andrew Howard, now at ISI, for configuring the simulation for our experiments. We are especially grateful for the significant financial support provided by the Advanced Engineering Centre for Manufacturing, a joint activity of the University of Melbourne and RMIT University.

\section{References}

1. P. Busetta, R. Rönnquist, A. Hodgson, A. Lucas: JACK Intelligent Agents - Components for Intelligent Agents in Java. Proceedings of the International Conference on Autonomous Agents (2001)

2. P. R. Cohen, A. Cheyer, M. Wang, S. C. Baeg: An open agent architecture. Proceedings of the AAAI Spring Symposium on Software Agents. (1994) 
3. A. Hodgson, R. Rönnquist, P. Busetta Specification of Coordinated Agent Behaviour (the SimpleTeam Approach). Technical Report, Agent Oriented Software Pty. Ltd. (2001)

4. A. Howard: MuCows. RoboCup-00: Robot Soccer World Cup IV Springer LNCS Vol. 2019 (2001)

5. L. E. Parker: Heterogeneous Multi-Robot Cooperation PhD thesis. Department of Electrical Engineering and Computer Science, Massachusetts Institute of Technology (1994)

6. P. Stone, M. Veloso: Task decomposition, dynamic role assignment, and lowbandwidth communication for real-time strategic teamwork. Artificial Intelligence, volume 100, number 2, (June, 1999)

7. D. Pynadath, M. Tambe: An automated teamwork infrastructure for heterogeneous software agents and humans. Journal of Autonomous Agents and Multi-agent Systems (2001)

8. J. Thomas, K. Yoshimura, A. Peel: RooBots. RoboCup-01: Robot Soccer World Cup V (2002)

9. K. Yoshimura, R. Rönnquist, E. Sonenberg: An approach to specifying coordinated agent behaviour. Proceedings of the Third Pacific Rim International Workshop on Multi-Agents (2000)

10. K. Yoshimura: Towards real-time strategic teamwork in robotic soccer. Master Thesis. The University of Melbourne (2002). http://www.cs.mu.oz.au/ kyosh/thesis/thesis.html 